\title{
Catastrophic complications from filler injection on external genitalia
}

\author{
Byeong Soo Kwon, Jin Woo Kim \\ Department of Plastic Surgery, Inje University Busan Paik Hospital, Busan, Korea
}

Soft tissue filler injections are widely used due to their immediate effects, predictable results, and high stability. However, as the use of soft tissue filler injections has increased, various complications have been reported. We report a life-threatening complication in a patient who developed sepsis and necrotizing fasciitis. A 45-year-old woman presented with right leg pain and discharge from the labia majora. The patient had received a soft tissue filler injection of unknown composition 1 year earlier and had recently undergone incision and drainage for an inflammatory cystic nodule. Antibiotic treatment was administered for cellulitis, but the infection progressed to necrotizing fasciitis and sepsis. Fasciotomy and intensive care unit treatment improved the systemic infection, but the soft tissue filler injection site did not respond to treatment for 1 month. Thus, the injection site was covered with a pedicled vertical rectus abdominis musculocutaneous flap after wide excision. The area of skin necrosis on the leg was covered with split-thickness skin grafts. Infections occurring after soft tissue filler injections are related to biofilms, and treatment is sometimes difficult. Therefore, although soft tissue filler injections have a favorable safety profile, it is important to be aware of the risk of life-threatening complications.

Keywords Genitalia / Fasciitis, necrotizing / Sepsis / Biofilms
Correspondence: Jin Woo Kim Department of Plastic Surgery, Inje University Busan Paik Hospital, 75 Bokji-ro, Busanjin-gu, Busan 47392, Korea

Tel: +82-51-890-6139

Fax: +82-51-890-6114

E-mail: jinooda@hanmail.net

Received: October 6, 2020 • Revised: November 4, 2020 - Accepted: November 10, 2020

pISSN: 2234-6163 • elSSN: 2234-6171 • https://doi.org/10.5999/aps.2020.01907• Arch Plast Surg 2021;48:10-14

\section{INTRODUCTION}

Several types of soft tissue filler injections have been developed and are widely used for augmentation in plastic surgery. Soft tissue fillers are advantageous because they have immediate clinical effects and predictable results. Their delivery is relatively simple, and the recovery time is short. Moreover, they are relatively safe [1]. Therefore, the number of soft tissue filler injections has increased over the past 20 years, reaching 2.7 million in 2019 in the United States [2]. The currently available types of soft tissue fillers include substances such as polymer gel, hyal- uronic acid, poly-L-lactic acid, and silicone [3].

Although soft tissue filler injections are widely used and considered relatively safe, they may also cause side effects including lumps, infection, swelling, and skin necrosis [4]. Most side effects are minor and self-limited, and are often treated with relatively simple methods, such as massage and medication [5-7]. However, rare cases of systemic symptoms have been reported. In particular, cases of life-threatening side effects such as sepsis and pulmonary embolism have been reported [4,8-12].

Since soft tissue injections have the potential to cause rare cases of life-threatening complications, it is very important for clini- 
cians to familiarize themselves with the possible side effects. For this reason, we report the case of an infection that occurred as a late complication after soft tissue filler injection. The infection involved the labia majora, and subsequently progressed to sepsis and necrotizing fasciitis that resulted in necrosis of the skin on the entire right leg.

\section{CASE}

A 45-year-old Asian woman with no underlying medical conditions was admitted to the emergency room with bilateral pain in the inguinal area, pus-like discharge, and pain and redness in the right thigh. The patient had received a filler injection of unknown composition into the labia majora and nearby soft tissues at a clinic 1 year prior to admission. Since then, the patient regularly experienced discomfort, with redness and swelling at the soft tissue filler injection site. Two to three months prior to admission, she noticed a palpable mass with intermittent pain. One week before the visit, the mass increased in size, with worsening pain and swelling. The patient underwent incision and drainage at a local clinic, but the redness, swelling, and discharge persisted. Wound healing was slow, and she started experiencing pain in the right leg approximately 2 days before admission. On physical examination, swelling and redness were noted around the postoperative wound, with pus-like discharge from both labia majora. In addition, there was redness, swelling, and with tenderness of the entire right thigh. The patient's vital signs were stable. She was diagnosed with soft tissue filler injection-associated cellulitis. Intravenous antibiotics were administered, and the patient was followed up for 2 days.

The patient developed fever and mild dyspnea on the second day of hospitalization. Her blood pressure, heart rate, respiratory rate, and temperature were 105/80 $\mathrm{mmHg}, 112$ beats $/ \mathrm{min}, 24$ breaths $/ \mathrm{min}$, and $38.2^{\circ} \mathrm{C}$, respectively. Laboratory markers of infection and inflammation also became rapidly elevated. Her white blood cell count, platelet count, C-reactive protein level, and erythrocyte sedimentation rate were $4,250 / \mu \mathrm{L}, 7,300 / \mu \mathrm{L}$, $42 \mathrm{mg} / \mathrm{dL}$, and $42 \mathrm{~mm} / \mathrm{hr}$, respectively. However, no bacteria were detected on culture of samples from the wound (Table 1).

A chest $\mathrm{X}$-ray showed signs of pulmonary edema, and magnet-

\section{Table 1. Initial evaluation of laboratory markers}

\begin{tabular}{lc}
\hline Parameter & Value \\
\hline White blood cell count $(/ \mu \mathrm{L})$ & 4,250 \\
Platelet count $(/ \mu \mathrm{L})$ & 7,300 \\
C-reactive protein level $(\mathrm{mg} / \mathrm{dL})$ & 42 \\
Erythrocyte sedimentation rate $(\mathrm{mm} / \mathrm{hr})$ & 42 \\
Initial bacterial culture (pus) & No bacteria
\end{tabular}

ic resonance imaging of the lower leg and pelvis showed signs of myositis at the right pectineus and adductor muscle group, as well as air bubbles with soft tissue swelling in the vulva area. The patient was diagnosed with sepsis secondary to cellulitis and myositis of the thigh. Intravenous antibiotics (vancomycin and piperacillin/tazobactam) were administered, and the patient was admitted to the intensive care unit (ICU). Corynebacterium striatum was subsequently identified in the follow-up wound culture.

After 1 week, the infection progressed to necrotizing fasciitis accompanied by skin necrosis (Fig. 1). Right leg fasciotomy and necrotic tissue debridement were performed, followed by regular wound debridement. Subsequently, the systemic infection was controlled through continuing ICU care, but the wound on both labia majora did not improve. As a result of necrosis, there was a skin defect spanning the entire right leg that required surgical treatment. The operation was performed under general anesthesia and entailed wide excisions to both labia majora and the pubis. Small spherical, transparent nodules, presumed to be filler particles, were observed in the excised tissues (Fig. 2). The

Fig. 1. Clinical photographs of necrotizing fasciitis

(A) Anterior proximal thigh region. (B) Anterior distal thigh region. (C) Pretibial region.
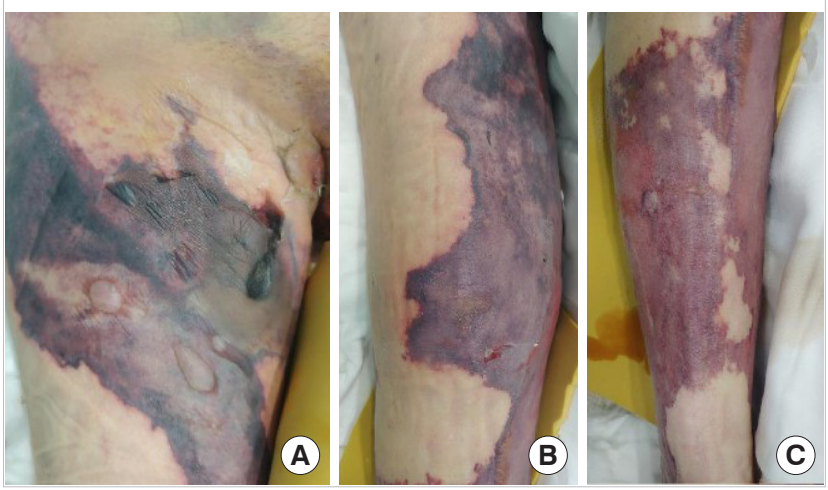

Fig. 2. Gross findings of the excised tissue

Multiple transparent spheres suspected to be filler particles were scattered throughout the excised tissue.

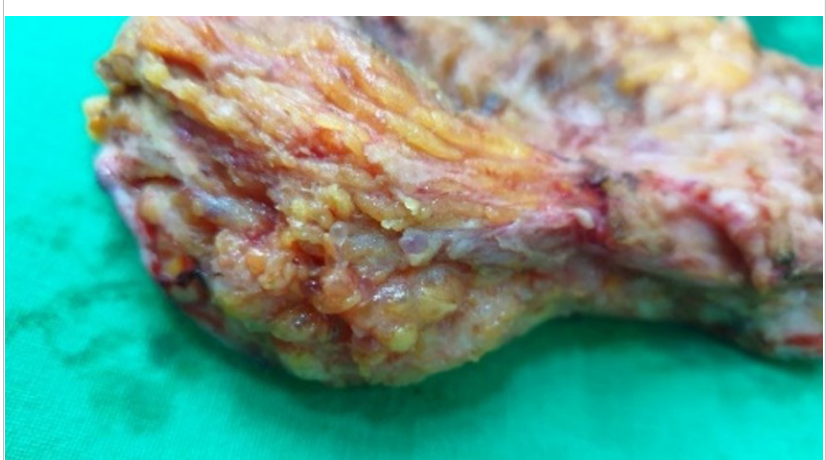


Fig. 3. Postoperative 3-month clinical photograph

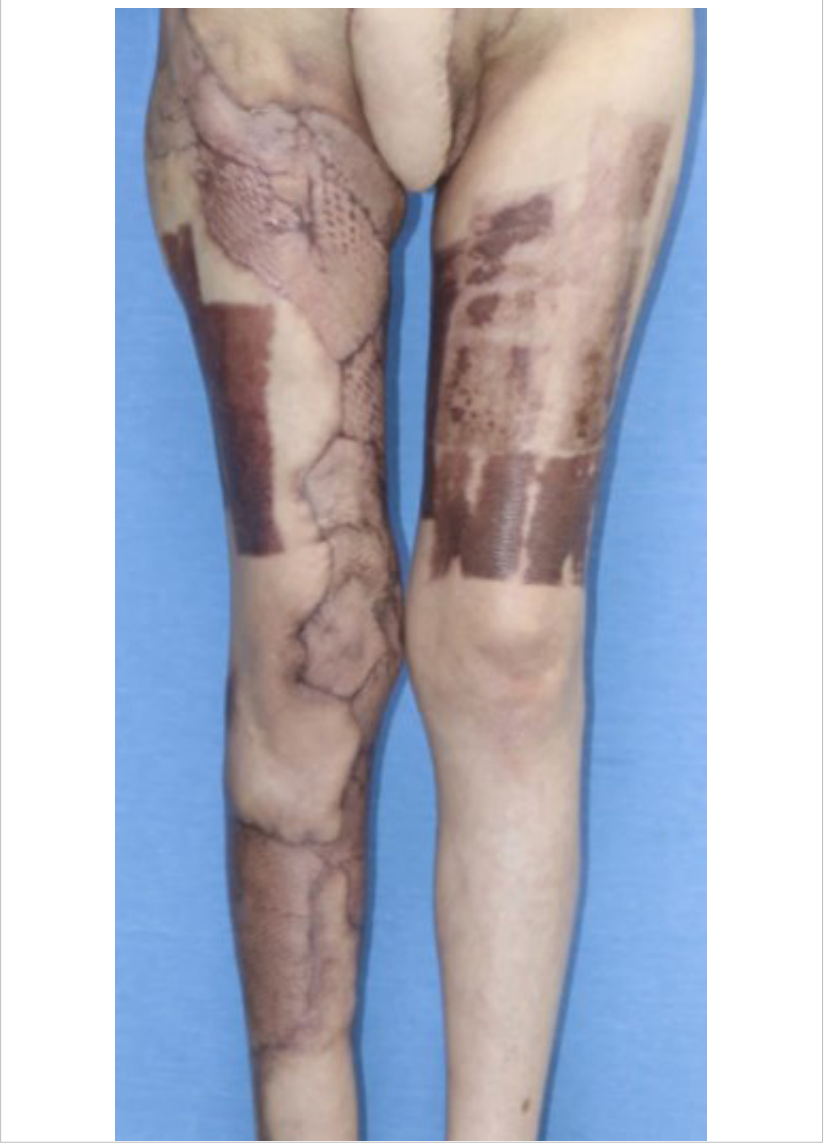

left labium majus, which had relatively minor tissue loss, was covered with a local flap, whereas the right labium majus, with relatively severe tissue loss, had a defect measuring $8 \times 8 \mathrm{~cm}$ and could not be covered by a local flap. Therefore, it was covered with a pedicled vertical rectus abdominis musculocutaneous flap. Split-thickness skin grafts were performed for the skin defect on the entire right lower limb. The wound healed after surgery, and the patient was discharged without any complications (Fig. 3).

\section{DISCUSSION}

Soft tissue filler injections, which are mainly used for soft tissue augmentation, have a favorable safety profile, rapid effects, and predictable effects compared to surgical treatment [1]. These characteristics caused the number of soft tissue filler injection procedures to increase dramatically, as exemplified by an increase in the United States from 650,000 in the 2000s to 2.7 million in 2019 [4]. As fillers became popular, various types of fillers were developed. Depending on a filler's duration of action, it can be classified as temporary or permanent. Temporary fillers include hyaluronic acid, poly-L-lactic acid, and calcium hydroxyapatite, whereas permanent fillers include silicone, polyacrylamide hydrogels, polymethylmethacrylate, and polyalkylimide [3]. Although soft tissue filler injection procedures are considered safe, various side effects have been reported as a result of the increasing variety in the content of fillers and the number of filler injection procedures [1-12].

The possible complications of soft tissue filler injections include both self-limited side effects (such as swelling, erythema, and ecchymosis) and rare, life-threatening side effects. Redmond et al. [9] reported a case of sepsis after filler injection and Yang et al. [8] reported a case of death due to pulmonary embolism caused by filler injection. We report a case of sepsis, which is a life-threatening complication of filler injection. Ortiz et al. [4] analyzed data from the Food and Drug Administration in the United States, and found that side effects leading to systemic symptoms accounted for only $0.9 \%$ of the side effects of soft tissue filler injection. However, $0.1 \%$ of all side effects are serious complications, including death. Therefore, it is important to be aware that even though filler injection is generally safe, lifethreatening complications can occur in extremely rare cases.

The side effects of soft tissue filler injections can be classified as early or late complications, depending on the time of occurrence. Early complications, which are more common, occur within 2 weeks of the soft tissue filler injection [6]. Early complications include erythema and swelling secondary to an individual's reaction to a foreign body and blood vessel occlusion. Late complications include nodule formation and foreign body granuloma $[6,7]$.

Some studies have reported that among patients with late complications, inflammatory nodules or swellings are associated with biofilms [13]. A biofilm is an aggregation of bacterial cells attached to an artificial surface, embedded in a matrix of extracellular bacterial macromolecules. Biofilms can form on any long-lasting filler [14]. Once a biofilm forms, culture and identification of the bacteria are difficult as the thickness of a biofilm layer is 5-1,200 $\mu \mathrm{m}$. This interferes with the collection of test samples and can cause false negative culture test results [15]. Furthermore, the passage of antibiotics through the biofilm layer is inhibited. This may lead to antibiotic resistance and evasion of the host's immune response [16]. For these reasons, biofilmrelated filler-associated infections may be difficult to treat. In our case, it can be assumed that a biofilm had formed as bacteria were not identified in the initial wound culture. Consequently, initial antibiotic treatment failed, and the condition progressed to sepsis and necrotizing fasciitis.

There is no established standard treatment for infection as a late complication of soft tissue injection. Since these infections involve bacteria, antibiotic treatment should be performed ini- 
tially. Antibiotics should be selected according to the results of bacterial culture and antibiotic susceptibility testing. However, biofilms might interfere with bacterial identification. For this reason, it may be difficult to identify the bacteria and to select proper antibiotics. Marusza et al. [13] recommended combination therapy with clarithromycin and moxifloxacin as empirical antibiotics for late bacterial infections. Clarithromycin is a macrolide antibiotic with bactericidal properties against Gram-positive and atypical bacteria. It has the effect of destroying biofilm structures. Moxifloxacin is a quinolone antibiotic with bactericidal properties against Gram-positive aerobic bacteria, Gramnegative aerobic bacteria, anaerobes, and atypical bacteria. It can penetrate the biofilm environment and affect bacteria in a biofilm [10]. In addition, Urdiales-Galvez et al. [5] recommended that since infection may be caused by an atypical organism, ethambutol or rifampicin should be used as an empirical antibiotic alongside clarithromycin. Therefore, it is recommended to administer antibiotic combination therapy with broad-spectrum antibiotics, including those capable of covering atypical bacteria. In addition, a common point of most studies is that infections associated with soft tissue fillers injection can lead to biofilm formation; therefore, it is important to remove all fillers with a biofilm $[5,10]$. As a result, it is necessary to consider surgical resection of the infected tissues, including the filler-injected site.

Even though filler injections are used in several parts of the body due to their stability, most life-threatening complications have been reported to involve pulmonary sequelae, including pulmonary embolism and alveolar hemorrhage after vaginal rejuvenation $[8,10-12]$. The vaginal wall is surrounded by a wide venous plexus; hence, there is the possibility of making an intravenous injection. In addition, the movement of muscles around the vaginal wall may cause filler droplets to migrate into the blood vessels [12]. In this mechanism, the intravenous filler may form emboli or may interact with fibrinogen, increasing the risk of complications [10]. Therefore, the vaginal area is a dangerous zone for soft tissue filler injections.

Our patient developed a systemic infection after a surgical intervention for an inflammatory nodule that occurred after a soft tissue filler injection. The exact mechanism cannot be identified; however, it is thought that the surgical intervention acted as a trigger for the spread of infection. Similar to our case, ElShafey [17] reported four cases of infection caused by puncture or injection at old polyacrylamide injection sites. In that article, it was reported that the complications in all cases were related to polyacrylamide, although the exact mechanism was not known. The author suggested that surgical interventions, such as punctures or injections, broke the barrier between the tissue and polyacrylamide foreign body, causing inflammation and infec- tion. The complication in our case can be explained by the same mechanism. Moreover, the filler had an unknown composition and could have contained polyacrylamide.

In our case, the question arose of whether the infection was a complication of a soft tissue filler injection or a complication of incision and drainage. An inflammatory nodule and pus-like discharge were observed at the time of the patient's visit to the local clinic. Thus, we think that the soft tissue filler injection was the direct cause. However, we cannot completely exclude the possibility that this complication was caused by incision and drainage performed to remove the inflammatory nodule. The two hypotheses are identical in that infection control was difficult, since a soft tissue filler injection-associated infection occurred at the injection site. Regardless of the direct cause, it is important to underscore that it was highly challenging to control the soft tissue injection-associated infection.

In rare cases, after a soft tissue filler injection, an infection can occur that does not respond to antibiotic treatment, with the potential to develop into a potentially fatal condition due to biofilm formation. Therefore, although soft tissue filler injections have a favorable safety profile, it is important to be aware of their potential to cause life-threatening complications.

\section{NOTES}

\section{Conflict of interest}

No potential conflict of interest relevant to this article was reported.

\section{Ethical approval}

The study was approved by the Institutional Review Board of Inje University Busan Paik Hospital (IRB No. 20-0174) and performed in accordance with the principles of the Declaration of Helsinki. Written informed consent was obtained.

\section{Patient consent}

The patient provided written informed consent for the publication and the use of her images.

\section{Author contribution}

Conceptualization: JW Kim. Methodology: BS Kwon. Project administration: JW Kim. Visualization: BS Kwon. Writing original draft: BS Kwon. Writing - review \& editing: JW Kim.

\section{ORCID}

Byeong Soo Kwon https://orcid.org/0000-0002-7550-3994 Jin Woo Kim https://orcid.org/0000-0002-7660-843X 


\section{REFERENCES}

1. Daines SM, Williams EF. Complications associated with injectable soft-tissue fillers: a 5-year retrospective review. JAMA Facial Plast Surg 2013;15:226-31.

2. American Society of Plastic Surgeons (ASPS). Plastic surgery statistics report [Internet]. Arlington Heights, IL: ASPS; c2020 [cited 2020 Oct 1]. Available from: https://www.plasticsurgery.org/documents/News/Statistics/2019/plasticsurgery-statistics-full-report-2019.pdf.

3. Ferneini EM, Beauvais D, Aronin SI. An overview of infections associated with soft tissue facial fillers: identification, prevention, and treatment. J Oral Maxillofac Surg 2017;75: 160-6.

4. Ortiz AE, Ahluwalia J, Song SS, et al. Analysis of U.S. Food and Drug Administration data on soft-tissue filler complications. Dermatol Surg 2020;46:958-61.

5. Urdiales-Galvez F, Delgado NE, Figueiredo V, et al. Treatment of soft tissue filler complications: expert consensus recommendations. Aesthetic Plast Surg 2018;42:498-510.

6. Sclafani AP, Fagien S. Treatment of injectable soft tissue filler complications. Dermatol Surg 2009;35 Suppl 2:1672-80.

7. Bailey SH, Cohen JL, Kenkel JM. Etiology, prevention, and treatment of dermal filler complications. Aesthet Surg J 2011; 31:110-21.

8. Yang Y, Sheng H, Gu Q et al. Death caused by vaginal injection of hyaluronic acid and collagen: a case report. Aesthet Surg J 2020;40:NP263-8.
9. Redmond E, Forde JC, Flood HD. Filling the void: a case of sepsis following the injection of a penile bulking agent. BMJ Case Rep 2014;2014:bcr2013203054.

10. Han SW, Park MJ, Lee SH. Hyaluronic acid-induced diffuse alveolar hemorrhage: unknown complication induced by a well-known injectable agent. Ann Transl Med 2019;7:13.

11. Park HJ, Jung KH, Kim SY, et al. Hyaluronic acid pulmonary embolism: a critical consequence of an illegal cosmetic vaginal procedure. Thorax 2010;65:360-1.

12. Duan Y, Zhang L, Li S, et al. Polyacrylamide hydrogel pulmonary embolism: a fatal consequence of an illegal cosmetic vaginal tightening procedure: a case report. Forensic Sci Int 2014;238:e6-10.

13. Marusza W, Olszanski R, Sierdzinski J, et al. Treatment of late bacterial infections resulting from soft-tissue filler injections. Infect Drug Resist 2019;12:469-80.

14. Rohrich RJ, Monheit G, Nguyen AT, et al. Soft-tissue filler complications: the important role of biofilms. Plast Reconstr Surg 2010;125:1250-6.

15. Hall-Stoodley L, Stoodley P, Kathju S, et al. Towards diagnostic guidelines for biofilm-associated infections. FEMS Immunol Med Microbiol 2012;65:127-45.

16. Singh S, Singh SK, Chowdhury I, et al. Understanding the mechanism of bacterial biofilms resistance to antimicrobial agents. Open Microbiol J 2017;11:53-62.

17. El-Shafey ESI. Complications from repeated injection or puncture of old polyacrylamide gel implant sites: case reports. Aesthetic Plast Surg 2008;32:162-5. 